'CESFAM Arrau Méndez, Departamento de Salud, I. Municipalidad Parral, Chile. ${ }^{2}$ CESFAM Dr. J.D. Astaburuaga, Departamento de Salud, I. Municipalidad de Talca, Chile. ${ }^{a}$ Kinesiólogo, Licenciado en Kinesiología.

bKinesiólogo, Magíster en Kinesiología.

Recibido el 27 de mayo de 2014, aceptado el 7 de abril de 2015 .

Correspondencia a: Gabriel Marzuca N. Dirección 6 y 7 oriente, 12 norte sin número, Talca, Chile. Teléfono-Fax: 071-635800 gmarzuca@gmail.com

\section{Criterios de valoración geriátrica integral en adultos mayores con dependencia moderada y severa en Centros de Atención Primaria en Chile}

\author{
CAROLINA ANDREA MUÑOZ SILVA ${ }^{1, a}$, PEDRO ANTONIO \\ ROJAS ORELLANA ${ }^{2}$, GABRIEL NASRI MARZUCA-NASSR ${ }^{2, b}$
}

\section{Functional geriatric assessment in primary health care}

\begin{abstract}
The functional assessment of moderately or severely dependent older people encompasses social, psychological and biological aspects that may influence their quality of life and their degree of independence. This paper reviews the global geriatric assessment that should be performed in primary health care (PHC) for moderately or severely dependent older people. Since 2012 in PHC in Chile, the norm establishes that the degree of independence of older people should be assessed using the Barthel scale and caregiver stress should be evaluated using Zarit scale. People with severe disability should receive home care. We recommend to evaluate also cognitive aspects using the minimental state examination (MMSE) to Barthel Index or using the Functional Independence Measure (FIM), since they are closely associated with functional capacity.
\end{abstract}

(Rev Med Chile 2015; 143: 612-618)

Key words: Dependency (Psychology); Frail elderly; Functionally-Impaired Elderly; Geriatric Assessment; Primary Health Care.
S e define envejecimiento como un "proceso multifactorial que tiene lugar en la última etapa del ciclo vital, duración máxima entre la concepción y la muerte, y que se caracteriza por la disminución progresiva de la capacidad funcional en todos los tejidos y órganos del cuerpo" ${ }^{\text {. }}$.

En Chile, se considera población envejecida a los 65 años de edad y en la Organización de las Naciones Unidas a los 60 años ${ }^{2}$. La esperanza de vida en la mayor parte de nuestra historia era de 20 a 50 años, pero a partir del siglo XX ha aumentado exponencialmente, generando un cambio en la pirámide poblacional disminuyendo su ancho en la base y aumentando en la cima ${ }^{1,3}$. Los cambios en los estilos de vida y nuevos tratamientos de salud, se encuentran entre las principales causas del aumento de las expectativas de vida ${ }^{4}$. Este cambio en la pirámide poblacional también afecta a Chile, donde el promedio de vida es alrededor de los 78,4 años y la supervivencia entre los 80 y 90 años es de $37 \%$ para las mujeres y $25 \%$ para los hombres, siendo el tercer país de Latinoamérica más envejecido y se espera que está población se incremente de $10,1 \%$ en el año 2001 a $19 \%$ para el $2025^{5,6}$. Este aumento del envejecimiento de la población, lleva a más casos de adultos mayores con dependencia moderada y severa. Realizar un abordaje correcto en esta población es de suma importancia, por lo tanto, el propósito de esta revisión es dar a conocer una valoración geriátrica integral para pacientes con dependencia moderada y severa en Atención Primaria de Salud (APS). 


\section{Alteraciones fisiológicas del reposo prolongado}

Los adultos mayores no fallecen por el envejecimiento, sino que por las complicaciones relacionadas a las enfermedades crónicas que tienen ${ }^{4}$. El reposo prolongado afecta a todos los sistemas corporales y mientras más tiempo involucre, más graves serán los efectos. Las complicaciones más importantes se pueden agrupar en tres esferas: físicas, cognitivas y sociales ${ }^{7}$.

Además de la Sarcopenia ${ }^{8,9}$, existe disminución de la fuerza muscular producida por el reposo prolongado. Esta disminuye entre $1 \%$ a $3 \%$ por día y de $10 \%$ a $15 \%$ por semana. Si este reposo se mantiene por dos o más meses, el músculo se atrofia llegando hasta a la mitad de su tamaño original $^{10}$.

La función cardiovascular también se altera, la fuerza contráctil del corazón disminuye a una tasa anual de 7,5\% después de los 30 años. Esta declinación llevará a una reducción significativa del volumen y de la cantidad de sangre expulsada durante la contracción ventricular, reducción en la capacidad periférica del flujo de sangre y en la superficie transversal arterial ${ }^{11}$.

En el sistema respiratorio se producen atelectasias, desaturación de oxígeno y predisposición a neumonías. Además, encontramos disminución de la capacidad vital, volumen corriente, capacidad máxima respiratoria y capacidad funcional residual $^{12}$.

En el esqueleto, la osteoporosis es la principal consecuencia provocada por una pérdida de masa ósea progresiva con la edad. Este debilitamiento puede provocar fracturas espontáneas, durante procedimientos del cuidado diario existiendo múltiples factores de riesgo incluyendo el reposo prolongado, enfermedades y desnutrición ${ }^{13,14}$.

La formación de cálculos renales, infecciones urinarias y cateterización crónica, son alteraciones frecuentes producidas a nivel renal por el reposo prolongado, además se producen trastornos gastrointestinales tales como pérdida del apetito, estreñimiento por disminución del peristaltismo e impactación o retención fecal ${ }^{7}$.

Con respecto al sistema cutáneo, se reduce la perfusión de sangre en las prominencias óseas, la disminución de la hidratación provoca piel seca y escamosa, hay pérdida de fibras elásticas y glándulas sebáceas, esto puede predisponer el desarrollo de las úlceras por presión (UPP) ${ }^{15}$.

\section{Envejecimiento, reposo prolongado y función cognitiva}

Uno de los primeros cambios producidos es la disminución de la masa del cerebro en relación al envejecimiento a una tasa de $5 \%$ de su peso durante cada década a partir de los $40 \operatorname{años}^{16}$. Las funciones cerebrales también se encuentran afectadas, principalmente la memoria, el aprendizaje, la recuperación de nueva información, solución de problemas, el procesamiento y la rapidez de respuesta ${ }^{17}$. La memoria se describe como un proceso mental a través del cual fijamos, retenemos y reproducimos todo aquello que alguna vez experimentamos. Siendo la pérdida de esta, una de las alteraciones más benignas y que se encuentra vinculado con el envejecimiento normal, presentándose en alrededor de 70\% de los adultos mayores $^{17,18}$. Con el envejecimiento se observa un enlentecimiento generalizado en el procesamiento de la información y una disminución en la capacidad de cambiar o alternar el foco de atención ${ }^{19}$. El deterioro cognitivo leve es una etapa de transición entre el envejecimiento normal y la demencia, siendo el punto final de la disminución de la función cognitiva y psicosocial la enfermedad de Alzheimer u otros procesos demenciantes ${ }^{20,21}$.

\section{Funcionalidad y dependencia}

La funcionalidad o independencia funcional "es la capacidad de cumplir acciones requeridas en el diario vivir, para mantener el cuerpo y subsistir independientemente" . Por otro lado, la dependencia se define como una "disminución o ausencia de la capacidad para realizar alguna actividad en la forma correcta o dentro de los márgenes considerados normales"22.

Los primeros instrumentos para evaluar los niveles de dependencia e independencia con respecto a las actividades de la vida diaria (AVD) se crearon en los años 70, estos son el Índice de Katz y el Îndice de Barthel. Actualmente, estos instrumentos no se aplican aislados y son incorporados dentro de una valoración geriátrica integral (VGI). La VGI determina un diagnóstico multifuncional que ayuda a identificar y cuantificar los problemas físicos, funcionales, psíquicos y sociales que puede presentar el adulto mayor, muchas veces no identificados en la historia clínica tradicional y que, además, ayuda a establecer un plan de seguimiento 
evolutivo. Dentro de los beneficios de la VGI encontramos una disminución en la mortalidad, disminución en las hospitalizaciones y mejoría del estado funcional, entre otras ${ }^{23}$. En este escrito, se entenderá VGI como una evaluación compuesta tanto por antecedentes personales, educacionales, médicos, sociodemográficos, funcionales y $\operatorname{cognitivos}^{5,24}$. Esta nos permite evaluar el déficit y las necesidades actuales de los adultos mayores dependientes, preparando un plan de tratamiento integral que incluye al cuidador, disminuyendo la institucionalización, hospitalizaciones y fallecimientos ${ }^{19}$.

\section{Valoración geriátrica integral para pacientes con dependencia moderada y severa en Atención Primaria de Salud en Chile}

En APS en Chile, el examen de medicina preventiva del adulto mayor (EMPAM) se realiza una vez al año a toda persona mayor de 65 años. Dentro del EMPAM se encuentra la aplicación del diagnóstico funcional del adulto mayor o EFAM, el cual permite detectar en forma integral los factores de riesgo que puede presentar el adulto mayor que es autovalente. El EFAM, basado en una parte $\mathrm{A}$ y $\mathrm{B}$, se realiza junto con la aplicación del minimental. La parte A, permite detectar si el sujeto es funcional o tiene algún grado de dependencia, la parte B apunta a evaluaciones del riesgo cardiovascular, estado cognitivo actual, depresión $\mathrm{y}$ ansiedad. Se debe tener en cuenta que la parte $\mathrm{B}$ no se aplica si el adulto mayor tiene algún nivel de dependencia en la parte $\mathrm{A}^{25}$. Estos últimos sujetos deben ser evaluados con el Î́ndice de Barthel.

Debido a lo anterior, es importante detectar aquellas situaciones que puedan colocar en riesgo la mantención del estado funcional, con la ayuda de instrumentos creados para este fin, por lo cual toda evaluación debe incluir factores cognitivos, motores, psicológicos, demográficos, educacionales, sociales y del cuidador ${ }^{5}$. A continuación se proponen consideraciones principales e instrumentos para realizar una VGI de un adulto mayor con dependencia moderada o severa en APS (Tabla 1).

\section{Identificación y clasificación de la persona con dependencia moderada o severa}

En la entrevista de ingreso, ya sea en el Centro de Salud o en domicilio, se deben preguntar datos personales, nivel educacional, datos socio demográficos, antecedentes médicos, patologías de base y tratamiento farmacológico. Se evalúan los signos vitales (frecuencia cardiaca y respiratoria, presión arterial, saturación de oxígeno) y la condición en la

Tabla 1. Resumen de los principales instrumentos de evaluación para realizar una valoración geriátrica integral a pacientes con dependencia moderada y severa

\begin{tabular}{|c|c|c|c|c|c|}
\hline & FIM & Índice Barthel & $\begin{array}{l}\text { Minimental } \\
\text { abreviado }\end{array}$ & Zarit & $\begin{array}{c}\text { Zarit } \\
\text { abreviado }\end{array}$ \\
\hline Objetivo & $\begin{array}{l}\text { Evaluar nivel de } \\
\text { independencia } \\
\text { mediante realiza- } \\
\text { ción de AVD }\end{array}$ & $\begin{array}{l}\text { Evaluar la } \\
\text { funcionalidad e } \\
\text { intensidad de la } \\
\text { asistencia dada } \\
\text { por el cuidador }\end{array}$ & $\begin{array}{l}\text { Evaluar deterioro } \\
\text { cognitivo }\end{array}$ & $\begin{array}{l}\text { Evaluar sobrecar- } \\
\text { ga del cuidador }\end{array}$ & $\begin{array}{l}\text { Evaluar sobrecar- } \\
\text { ga del cuidador }\end{array}$ \\
\hline $\begin{array}{l}\text { Tiempo de aplicación } \\
\text { aproximado }\end{array}$ & $45 \min$ & $5 \mathrm{~min}$ & $10 \mathrm{~min}$ & $10 \mathrm{~min}$ & $3 \mathrm{~min}$ \\
\hline Sensibilidad & Alta sensibilidad & $\begin{array}{l}\text { Escasa sensibili- } \\
\text { dad en cambios } \\
\text { pequeños }\end{array}$ & $\begin{array}{l}89 \% \text { en adultos } \\
\text { mayores }\end{array}$ & $93 \%$ & $100 \%$ \\
\hline Consistencia interna & $\begin{array}{l}\text { Total } \\
0,88-0,97 \\
\text { Motor } \\
0,86-0,98 \\
\text { Cognitivo } \\
0,68-0,96\end{array}$ & $0,86-0,92$ & $0,82-0,84$ & 0,91 & 0,84 \\
\hline
\end{tabular}

FIM: Functional Independence Measure; AVD: actividades de la vida diaria. 
cual se encuentra el adulto mayor al momento de la visita (estado de conciencia, estado nutricional, presencia de UPP, ubicación habitual del paciente, entre otras $)^{26}$. Luego, se recomienda la evaluación de la funcionalidad y asistencia dada por el cuidador con la Medida de Independencia Funcional (FIM), nivel de independencia respecto a las AVD con el Índice de Barthel, estado cognitivo con el minimental abreviado (MMSE) y, finalmente, la escala de sobrecarga del cuidador de Zarit.

\section{Medida de Independencia Funcional (FIM)}

El FIM es uno de los instrumentos más utilizados para realizar evaluación funcional en rehabilitación ${ }^{27}$. Se desarrolló entre 1984 y 1987 por la American Congress of Rehabilitation Medicine y la American Academy of Physical Medicine and Rehabilitation, evaluando la funcionalidad del paciente e intensidad de la asistencia dada por el cuidador $^{27,28}$.

Evalúa 18 AVD, asignándole un puntaje de 7 a 1 a cada una, entregando valores totales de 18 (asistencia total) a 126 puntos (independencia completa). Las actividades del FIM pueden agruparse en FIM motor de autocuidado (alimentación, arreglo personal, baño, vestido hemicuerpo superior, vestido hemicuerpo inferior, aseo perineal), control de esfínteres (vejiga e intestino), transferencias (desde y hacia a silla o silla de ruedas, traslado en baño, bañera o ducha) y locomoción (caminar o desplazarse en silla de ruedas, subir o bajar escaleras). El FIM cognitivo evalúa comunicación (comprensión, expresión) y conocimiento social (interacción social, solución de problemas y memoria $)^{27,28}$.

La confiabilidad del FIM depende del entrenamiento del evaluador en el uso del instrumento, principalmente en el ítem cognitivo, aunque al ser sometidos a entrenamiento, el nivel de confiabilidad es alto en el ítem cognitivo y aumenta levemente en el ítem motor. Presenta validez clínica, concordancia entre evaluadores, fiabilidad test-retest e intraobservador, pero falla la fiabilidad entre observadores en los ítems transferencias, locomoción, interacción social y cognitivo ${ }^{27-29}$.

\section{Índice de Barthel}

El índice de Barthel es uno de los instrumentos ampliamente utilizado para evaluar las AVD y cuantificarlas a través de su nivel de independencia. Se comenzó a aplicar en 1955 por Mahoney y Barthel, para medir la evolución de sujetos con procesos neuromusculares y musculoesqueléticos en un hospital para enfermos crónicos de Maryland ${ }^{30,31}$. Se define como una "medida genérica que valora el nivel de independencia del paciente con respecto a la realización de algunas actividades de la vida diaria"31.

Consta de diez ítems fundamentales de las AVD: alimentación, aseo personal, vestirse, arreglarse, deposición, micción, uso de retrete, traslados, deambulación y subir escaleras. La puntuación de cada actividad es diferente, asignándose un puntaje de $0,5,10$ o 15 puntos y la valoración total va desde los 0 (dependencia severa total) a 100 puntos (independencia) ${ }^{31,33}$.

Inicialmente se aplicaba a través de la observación, pero actualmente se utiliza la obtención de la información de forma directa del sujeto o de su cuidador principal (ambos métodos ofrecen una fiabilidad similar). Es de fácil aplicación e interpretación, posee buena aceptación de los pacientes, puede ser repetido periódicamente y es de rápida adaptación cultural ${ }^{34}$. Posee alta fiabilidad tanto inter e intraobservador. Es sensible para detectar progresos y/o deterioros en ciertas AVD, aunque tiene limitada sensibilidad a los cambios en niveles funcionales extremos ${ }^{31,34}$.

\section{Minimental abreviado}

Desarrollado por Folstein en 1975 como un "método práctico que permite establecer el grado del estado cognoscitivo del paciente y poder detectar demencia o delirio" 35,36 . Es una herramienta portátil, rápida y fácil de aplicar ${ }^{11,37}$. Consta de 11 ítems, concentrándose sólo en los aspectos cognitivos como orientación temporal y espacial, recuerdo inmediato y diferido de 3 palabras, atención o cálculo, nominación de 2 objetos, repetición de una frase, comprensión de una orden verbal y una escrita, escritura de una oración y copia de un diagrama. Los puntajes parciales son sumados, lográndose un máximo de 30 puntos, con una puntuación sobre 27 puntos como normal y menor a 24 puntos como sospecha patológica (12-24 puntos deterioro y 9-12 demencia) $)^{35,37}$.

González-Hernández et al. (2009), indicaron la influencia del factor educacional, siendo estadísticamente significativo y con diferencias de hasta 7 puntos entre los grupos con mayor y menor años de escolaridad. Quiroga et al. (2004), determinaron que la educación fue tan importante que 
entregó una especificidad de $44 \%$ del test ${ }^{37,38}$. En Chile fue modificado, abreviado y validado para la Encuesta Salud, Bienestar y Envejecimiento (SABE) en 1999, con el fin de eliminar el sesgo entregado por el nivel educacional. Presenta un puntaje de 19 puntos, donde un puntaje menor o igual a 13 puntos se considera alterado ${ }^{39}$. Ha sido validado por distintos países, desarrollándose incluso versiones para no videntes, hipoacúsicos y evaluaciones telefónicas ${ }^{39}$.

\section{Escala de sobrecarga del cuidador de Zarit}

Los pacientes dependientes moderados y severos, necesitan el apoyo de sus familias y/o cuidadores. Definiendo a este último como la persona que convive y mantiene la responsabilidad de proveer recursos a un paciente incapaz de sustentarse y están familiarizados con la situación médica y/o social del paciente ${ }^{40,41}$. Con la finalidad de evaluar a los cuidadores de los pacientes dependientes, se introduce el término de carga o sobrecarga del cuidador, siendo esta un estado psicológico de la combinación de problemas físicos, psicológicos o emocionales, sociales y financieros que se pueden experimentar al cuidar a los pacientes mayores. La sobrecarga objetiva se debe al desempeño de las tareas como asear al enfermo, darle de comer, movilizarlo y, subjetiva, se refiere a los sentimientos y emociones que provocan la labor del cuidado ${ }^{40,42}$.

Es uno de los instrumentos más utilizados a nivel internacional para evaluar la sobrecarga de cuidadores $^{41,43}$. Consta de 22 preguntas con 5 opciones cada una (puntaje de 0-4), cuyos resultados se suman y dan un puntaje total de 22 a 110 puntos. Este resultado clasifica al cuidador en ausencia de sobrecarga, sobrecarga ligera o sobrecarga intensa ${ }^{42}$.

Fue adaptada y validada al español en 1996, siendo revalidada en el año 2010. Presenta confiabilidad y consistencia, además ha demostrado validez de apariencia, contenido y detección de patologías principalmente de salud mental. Su principal inconveniente es su extensión, por lo que en España se ha validado recientemente una forma abreviada (Escala de Zarit Abreviada-EZA), la cual consta de 7 preguntas tomadas de la escala original, con 5 puntos cada una. En Chile, Breinbauer et al. (2009), validó la escala original y abreviada, presentando gran consistencia interna, confiabilidad test-retest tanto es su escala original como abreviada ${ }^{41,43}$.

\section{Atención domiciliaria a personas con dependencia severa en Chile}

El cuidado domiciliario de personas con discapacidad severa fue un importante vacío detectado por los equipos de salud del país, por esto, en el año 2006, el Ministerio de Salud (MINSAL) de Chile creó el "Programa de Atención Domiciliaria a Personas con Dependencia Severa”, con el objetivo de mejorar el cuidado de la persona postrada y aliviar a la familia, disminuyendo la carga del cuidador e impidiendo la institucionalización del paciente. Esto se desarrolla a través de atención al domicilio por un equipo multidisciplinario del centro de salud al paciente dependiente severo. Además, considera capacitación al cuidador y apoyo monetario (estipendio) a los cuidadores de pacientes que califican en esta condición ${ }^{26}$.

Dentro de las evaluaciones realizadas a los pacientes según la Norma de cuidados domiciliarios a personas que sufren de discapacidad severa, encontramos la escala de sobrecarga del cuidador de Zarit y hasta el año 2011, el Índice de Katz, excluyendo algún instrumento para evaluar el área cognitiva. A partir del año 2012 este programa en conjunto con el Programa del adulto mayor han determinado que el instrumento para medir la severidad de la dependencia en APS será el Índice de Barthel ${ }^{26}$. Nuestros resultados clínicos verifican una importante correlación entre FIM motor con el Índice de Barthel; MMSE con FIM cognitivo; nivel educacional y MMSE en pacientes dependientes moderados y severos ${ }^{44}$. Debido a lo mencionado anteriormente y a la literatura existente, se recomienda incorporar siempre la evaluación del área cognitiva, ya que se encuentra fuertemente ligada a la funcionalidad, agregando el MMSE al Barthel o utilizando el FIM.

Dentro de nuestra experiencia clínica en conjunto con la de algunos equipos de trabajo multidisciplinarios nacionales e internacionales, la aplicación del FIM es la que presenta mayor complejidad, ya que el evaluador debe ser sometido a un mayor entrenamiento antes de su aplicación y el tiempo en realizarlo es extenso al compararse con otros instrumentos, lo cual dificulta su uso en APS debido al escaso tiempo que uno posee para evaluar cada paciente.

En los centros de APS de Chile, se realiza énfasis en evaluar a los adultos mayores según el EMPAM de forma anual, el cual diagnosticará al adulto ma- 
yor como autovalente con riesgo, autovalente sin riesgo o riesgo de dependencia. Cuando un adulto mayor llega a realizarse el EMPAM, pero usen ayuda técnica u órtesis, sean personas mayores que necesitan asistencia de otra persona para recibir la atención del equipo de salud y/o personas mayores con discapacidad psíquica se debe realizar el índice de Barthel e ingresar al Programa de atención domiciliar para dependientes severos si cumple con los requisitos. Una vez ingresado en este programa, el adulto mayor tendrá las evaluaciones según la Norma de cuidados domiciliarios para personas con discapacidad severa (Barthel y Zarit), a pesar de esto, nosotros recomendamos incorporar siempre la evaluación del estado cognitivo para estos pacientes. Por otro lado, a pesar que esta norma de visita domiciliaria es para dependientes severos, se recomienda mantener estas evaluaciones también a los pacientes con dependencia moderada, en donde la evaluación del estado cognitivo cobra mayor importancia debido a su influencia en la funcionalidad.

\section{Conclusión}

Dentro de la batería de instrumentos para realizar una VGI a los adultos mayores con dependencia moderada y severa, el FIM es uno de los más utilizados a nivel internacional, ya que evalúa la funcionalidad de cada adulto mayor y la asistencia dada a éste por una tercera persona o cuidador, lamentablemente presenta inconvenientes: el evaluador necesita un mayor entrenamiento y requiere un gran tiempo de aplicación. La importancia de realizar una VGI en los adultos mayores con dependencia moderada y severa, nos permite vislumbrar la condición global del sujeto. Recomendamos incorporar siempre la evaluación del área cognitiva, ya que se encuentra fuertemente ligada a la funcionalidad, agregando el MMSE al Barthel o utilizando el FIM.

\section{Referencias}

1. Bernis C. Envejecimiento, poblaciones envejecidas y personas ancianas. Antropo 2004; 6: 1-14.

2. Albala C, Araya A, Barnett C, Borzuztku A, Bonout D, Castro S, et al. Situación demográfica en Chile. Manual Geriatría y Gerontología (Revista electrónica). 2000 (accedido el 01 de febrero de 2012). Disponible en:
http://escuela.med.puc.cl/publ/manualgeriatria/PDF/ Demografia.pdf.

3. Christensen K, Doblhammer G, Rau R, Vaupel J. Ageing population: the challenges ahead. Lancet 2009; 374: 1196-208.

4. Pérez V, Sierra F. Biología del envejecimiento. Rev Med Chile 2009; 137: 296-302.

5. Sanhueza M, Castro M, Merino J. Adultos mayores funcionales: Un nuevo concepto en salud. Ciencia y Enfermería 2005; 11 (2): 17-21.

6. Servicio Nacional del Adulto Mayor. Chile. Chile quiere a sus mayores. 2011 (accedido el 20 de enero de 2012). Disponible en: www.senama.cl/filesapp/cuentapublica2011.pdf.

7. Rousseau P. Clinical Review. Immobility in the Aged. Arch Fam Med 1993; 2: 169-77.

8. Roubenoff R, Hughes V. Sarcopenia: Current Concepts. J Gerontol A Biol Sci Med Sci 2000; 55 (12): 716-24.

9. Cruz-Jentoft A, Baeyens J, Bauer J, Boirie Y, Cederholm T, Landi F, et al. Sarcopenia: European Consensus on Definition and Diagnosis. Age and Ageing. 2010; 39: 412-23.

10. Molina J. Sarcopenia en la pérdida funcional: rol del ejercicio. Revista Hospital Clínico Universidad de Chile 2008; 19: 302-8.

11. Hyman B, Oden G, Wagner M. The aging process: Physiological changes and implications for educators and practitioners. J Adaptation \& Aging 2010; 34: 148-53.

12. Timiras P. Physiological basis of aging and geriatrics. Medicine and Science in Sports and Exercise 2003; 39 (8): 1435-45.

13. Olmos J, Martínez J, González J. Envejecimiento Músculo-Esquelético. REEMO 2007; 16 (1): 1-7.

14. Takamoto S, Saeki S, Yabumoto Y, Masaki H, Onishi T, Morimoto, et al. Spontaneous Fractures of Long Bones Associated With Joint Contractures In Bedridden Elderly Inpatients: Clinical Features And Outcome. JAGS 2005; 53 (8): 1439-41.

15. Lindreng M, Unosson M, Fredrikson M. Immobility-a major risk factor for development of pressure ulcers among adult hospitalized patients. J Caring Sci 2004; 18: 57-64.

16. Salech F, Jara R, Michea L. Cambios fisiológicos asociados al envejecimiento. Revista Médica Clínica Las Condes 2012; 23 (1): 19-29.

17. Bentosela M, Mustaca A. Efectos cognitivos y emocionales del envejecimiento: Aportes de investigaciones básicas para las estrategias de rehabilitación. Interdisciplinaria 2005; 22 (2): 211-35.

18. Casanova P, Casanova P, Casanova C. Deterioro cogniti- 
vo en la tercera edad. Revista Cubana de Medicina General Integral (Revista electrónica) 2008 (accedido el 02 de abril de 2012). Disponible en: http://scielo.sld.cu/scielo. php?script=sci_arttext\&pid=S0864-21252004000500012.

19. Marín P. Fragilidad en el Adulto Mayor y Valoración Geriátrica Integral. Reumatología 2004; 20 (2): 54-7.

20. Burns A, Zaudig M. Mild cognitive impairment in older people. The Lancet 2002; 360: 1963-5.

21. Pérez V. El deterioro cognitivo: Una mirada previsora. Revista Cubana de Medicina General Integral 2005; 21 (1-2).

22. Servicio Nacional del Adulto Mayor. Chile. Estudio Nacional de la Dependencia en las Personas Mayores. Santiago; 2009.

23. Redin JM. Valoración geriátrica integral (I). Evaluación del paciente geriátrico y concepto de fragilidad. SnalesSis San Navarra 1999; 22 (1): 41-50.

24. Reig A, Cabrero J, Lizán L. La valoración de la capacidad funcional, el bienestar psicológico y la salud mental en la atención primaria de salud. Atención Primaria. 2009; 41 (9): 515-9.

25. Ministerio de Salud. Chile. Manual de aplicación del examen de medicina preventivo del adulto mayor. (accedido en agosto de 2014). Disponible en http://web.minsal.cl/portal/url/item/ab1f81f43ef0c2a6e04001011e011907.pdf

26. Ministerio de Salud. Chile. Norma de cuidados domiciliarios de personas que sufren de discapacidad severa. Santiago; 2006. (accedido en agosto de 2014). Disponible en https://www.ssmaule.cl/paginas/index. php?option $=$ com_docman $\&$ task $=$ doc_download \&gi$\mathrm{d}=2154 \&$ Itemid $=123$

27. Wright J. The FIM (TM). The Center for Outcome Measurement in Brain Injury. (Revista electrónica) 2000 (accedido el 01 de febrero de 2012). Disponible en: http://www.tbims.org/combi/FIM.

28. Paolinelli C, González P, Doniez M, Donoso T, Salinas V. Instrumento de evaluación funcional de la discapacidad en rehabilitación. Estudio de confiabilidad y experiencia clínica con el uso del Functional Independence Measure. Revista Médica de Chile (Revista electrónica) 2001 (accedido el 10 de enero de 2012). Disponible en: http://www.scielo.cl/scielo.php?script=sci_arttext\&pi$\mathrm{d}=$ S0034-98872001000100004.

29. Mirallas J, Real M. ¿Índice de Barthel o Medida de Independencia Funcional? Rehabilitación 2003; 37 (3): 152-7.

30. Hsueh I, Lee M, Hsieh C. Psychometric characteristics of The Barthel Activities of Daily Living Index in stroke patients. J Formos Med Assoc 2001; 100: 526-32.

31. Barrero C, García S, Ojeda A. Índice de Barthel (IB): Un instrumento esencial para la evaluación funcional y la rehabilitación. Revista Plasticidad y Restauración Neurológica 2005; 4 (1-2): 81-5.

32. Fontana C, Estany J, Pujol J, Segarra I, Jordán Y. Concordancia entre índices de dependencia en las actividades de la vida diaria. Experiencia de aplicación en población geriátrica de ámbito rural. Enfermería Clínica 2002; 12 (2): 47-53.

33. Hsueh I, Lin J, Jeng J, Hseih C. Comparison of the psychometric characteristics of the functional independence measure, 5 item Barthel index, and 10 item Barthel index in patients with stroke. J Neurol Neurosurg Psychiatry 2002; 73: 188-90.

34. Trigas M, Ferreira L, Meijide H. Escalas de valoración funcional en el anciano. Galicia Clínica 2011; 72 (1): 11-6.

35. Folstein M, Folstein S, McHug P. "Mini-Mental State" A practical method for grading the cognitive state of patients fot the clinician. J Psychiat Res 1975; 12: 189-98.

36. Lee T, Chan J. Factores que afectan el estado cognitivo de personas que sufren de epilepsia. Revista de Neurología 2002; 34 (9): 861-5.

37. González-Hernández J, Aguilar L, Oporto S, Araneda L, Vásquez M, Von Bernhardi R. Normalización del "Mini-Mental State Examination" según edad y educación, para la población de Santiago de Chile. Revista Memoriza 2009; 3: 23-34.

38. Quiroga P, Albala C, Klaasen G. Validación de un test de tamizaje para el diagnóstico de demencia asociada a edad en Chile. Rev Med Chile 2004; 134: 467-78.

39. Organización Panamericana de Salud (OPS). Encuesta multicéntrica Salud Bienestar y Envejecimiento (SABE) en América Latina y el Caribe. Washington; 2001.

40. Gort A, Mingot M, Gómez X, Soler T, Torres G, Sacristan, et al. Use of the Zarit Scale for assessing caregiver burden and collapse in caregiving at home in dementias. International Journal of Geriatric Psychiatry 2007; 22: 957-62.

41. Breinbauer H, Vásquez H, Mayanz S, Guerra C, Millán T. Validación en Chile de la Escala de Sobrecarga del cuidador de Zarit en sus versiones original y abreviada. Rev Med Chile 2009; 13: 657-65.

42. Roig M, Abengozar C, Serra E. La sobrecarga en los cuidadores principales de enfermos de Alzheimer. Anales de Psicología 1998; 14 (2): 215-27.

43. Martínez-Carrasco M, Otermin P, Pérez-Camo V, Pujol J, Martín M, Gobartt A, et al. EDUCA study: Psychometric properties of the Spanish version of the Zarit Caregiver Buerden Scale. Aging \& Mental Health 2010; 14 (6): 705-11.

44. Muñoz C, Rojas P, Marzuca G. Valoración del estado funcional de adultos mayores con dependencia moderada y severa pertenecientes a un centro de salud familiar. Fisioter Pesqui 2015; 22 (1): 76-83. 